\title{
Schweizerische Gesellschaft für Plastische, Rekonstruktive und Ästhetische Chirurgie (SGPRAC)
}

\author{
Pierre Schertenleib ${ }^{a}$, \\ Catherine Perrin ${ }^{b}$ \\ a Dr. med., Präsident \\ b Dr. phil., Geschäftsführerin
}

\section{Verdan C. Histoire de la Société suisse de Chirurgie plastique, reconstructive et esthétique; 1985.}

\section{Korrespondenz:}

Catherine Perrin, PhD Office SGRPAC-SSCPRE 15 , avenue des Planches CH-1820 Montreux

info@plastic-surgery.ch www.plastic-surgery.ch

\section{Gründung und Zweck}

Die Schweizerische Gesellschaft für Plastische, Rekonstruktive und Ästhetische Chirurgie (SGPRAC) ist der Berufsverband der Fachärzte für Plastische, Rekonstruktive und Ästhetische Chirurgie. Sie wurde 1965 in Locarno gegründet. Die Gründungsmitglieder waren Federico Andina (Präsident), Rodolphe Meyer, HansUeli Buff, Claude Koechlin und Peter Ricklin [1]. Die Gesellschaft bezweckt die Förderung ihres Fachgebietes in Wissenschaft, Lehre und Forschung und pflegt Beziehungen zu verwandten Gesellschaften und Organisationen im In- und Ausland. Sie ist zuständig für die Wahrung der beruflichen Interessen sowie der ethischen Grundprinzipien des ärztlichen Handelns ihrer Mitglieder. Sie stützt sich dabei auf die Standesordnung der FMH und anerkennt deren Statuten. Sie ist zudem Mitglied der fmCH, dem Dachverband der chirurgischen und invasiven Fachgesellschaften.

Die SGPRAC ist zudem für die Weiter- und Fortbildung zuständig, indem sie die Inhalte der entsprechenden Programme definiert und deren Umsetzung mitgestaltet. Einmal pro Jahr findet ein wissenschaftlicher Kongress statt, in der Regel in der ersten Oktoberwoche, der anteilsmässig die drei Gebiete (plastische, rekonstruktive und ästhetische sowie Hand-Chirurgie) abdeckt. Die in Weiterbildung stehenden Assistenten haben zudem die Möglichkeit, ihre Forschungsresultate vorzustellen. Die Gesellschaft zählt 200 Mitglieder, davon 133 ordentliche. Die Gesellschaft wächst jedes Jahr im Durchschnitt um fünf Mitglieder.

\section{Qualitätssicherung}

Seit einigen Jahren legt die Gesellschaft besonderen Wert auf die Qualitätssicherung. Sie hat eigene Aufklärungsprotokolle erstellt, die es dem Arzt erlauben, Patienten eingehend und umfassend über den bevorstehenden Eingriff und die damit verbundenen Risiken zu informieren. Ab Juli 2009 werden erstmals die Brustimplantate in einem nationalen Register aufgezeichnet. Zum selben Zeitpunkt werden die Mitglieder eingeladen, die wichtigsten Operationen zu registrieren. Diese statistischen Angaben werden in einem ersten Schritt ein numerisches Benchmarking erlauben. Vor-

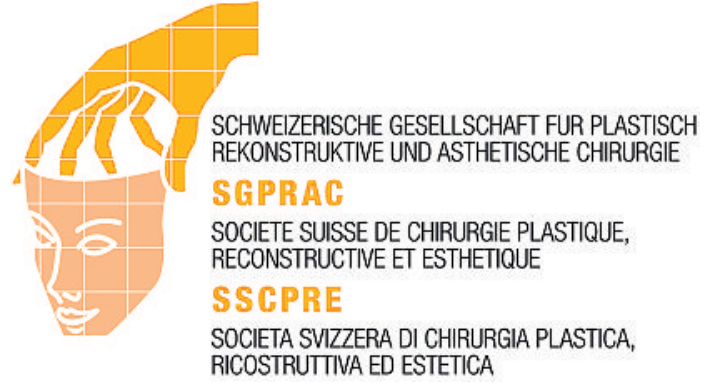

gesehen ist, dass in einem bis zwei Jahren nicht nur die Eingriffe registriert werden, sondern auch deren Verlauf (Revisionen, Komplikationen, Infektionen, usw.). Die Benchmark wird dann nicht nur numerisch sein, sondern auch klare Rückschlüsse betreffend Qualität des Eingriffs, Diagnose und Nachbehandlung erlauben. Diese Qualitätsinstrumente dienen nicht zum Selbstzweck, sondern sind ein erster Schritt hin zur Patientensicherheit und zur Hinterfragung des ärztlichen Handelns eines jeden Mitglieds der Gesellschaft.

\section{Information}

Seitdem die Ästhetische Chirurgie auch in der Schweiz einen sogenannten Boom erlebt, ist es der Gesellschaft ein grosses Anliegen, die Bevölkerung über die Risiken solcher Eingriffe eingehend zu informieren. Mit dem Aufschalten der neuen Homepage (www.plasticsurgery.ch) wurde ein grosses Informationsdefizit aus der Welt geschaft. Sie gibt umfassend Auskunft über das Gebiet und einen Überblick über die verschiedenen Eingriffe. Sie warnt auch vor Billigangeboten und Operationen im Ausland. Die Homepage enthält zudem die Liste aller aktiven Plastischen Chirurgen, die Mitglied in der Gesellschaft sind. Als weiteres Informationsinstrument hat die Gesellschaft eine Patientenbroschüre «Alles Wissenswerte über Plastische, Rekonstruktive und Ästhetische Chirurgie» herausgegeben, einen sogenannten Ratgeber, der unter www.plasticsurgery.ch heruntergeladen oder kostenlos bestellt werden kann (info@plastic-surgery.ch - oder 021 96321 39). Sei es die Homepage, sei es die Broschüre, beide erfreuen sich grosser Beliebtheit und werden rege benutzt. 